\title{
Research on Planning Model and Influence Factors of Transmission Line Online Monitoring Communication Network
}

\author{
Bin $\mathrm{Yu}^{1, \mathrm{a}}$, Xianggen Yin ${ }^{1, \mathrm{~b}}, \mathrm{Xu}$ Chen ${ }^{3, \mathrm{c}}$, Zhe Zhang ${ }^{4, \mathrm{~d}}$, and Jinmu Lai, e \\ ${ }^{1}$ State Key Laboratory of Advanced Electromagnetic Engineering and Technology, \\ Huazhong University of Science and Technology, Wuhan, China \\ arubean@qq.com, ${ }^{b} x g y i n @ h u s t . e d u . c n,{ }^{c}$ chenxu_hust@qq.com, ${ }^{d} z z$ mail2002@163.com, \\ Iaijinmu@126.com
}

\begin{abstract}
Keywords: Overhead transmission line; WSN; Communication network; Decision making; Fuzzy entropy weight.

Abstract. In this paper, a composite communication network is designed to solve the problems of bandwidth bottleneck and high latency in chain-type wireless communication network for transmission line online monitoring. A theoretical planning model of optical fiber separation placement is formulated with object of cost and latency optimization while respecting bandwidth constraint. PSO and fuzzy entropy weight based decision making method are used to solve the model and determine the most satisfactory planning scheme. Finally, we study the impact of the subjective weight, the data rate and network scale on decision making.
\end{abstract}

\section{Introduction}

The establishment of smart grid asks for intelligent management of transmission lines, which prompts transmission line online monitoring system to be informationized, networking and automatic. Thus, a large amount of sensors are deployed in transmission corridor and generate massive amount of data periodically [1]. Transmission towers span hundreds of miles and form a chain-type network. In chain-type wireless communication network, there are problems of bandwidth bottleneck of "the last mile" and high latency [2]. It is challenging for wireless network design to support speedy and extensive traffic requirement in smart grid. Nevertheless, with the rapid development of smart grid integration, the advanced applications such as short-term dynamic line rating [3] and operation risk assessment [4] place stringent requirements on communication network like fast delivery of enormous amount of data.

Therefore, there is an impending need to establish a high-performance data communication network that supports advanced operational requirements like real-time monitoring and fast control response. Combining with other communication technology of high-speed, low-latency and expensive, hybrid hierarchical network that spans wired, wireless and cellular technologies to provide cost effective or speedy communication are proposed in current researches. However, they are confined to one objective of cost or end-to-end latency $[5,6]$ so that economy and real-time of communication network can't be optimized in the meantime. With the wide application of Optical Fiber Composite Overhead Ground Wire (OPGW), in this paper, we plan to construct a composite communication network involving WSN and OPGW. In view of expensive arrangement cost of optical fiber separation, a theoretical planning model for optimal placement of optical fiber separated towers is formulated with objectives of minimizing cost and end-to-end latency while respecting bandwidth constraint. The influence factors of placement are also analyzed.

\section{Composite Communication Network Design and Planning Formulation}

A. Composite Communication Network Design

Fig. 1 shows our proposed composite framework. In order to meet newer requirements based on emerging smart grid applications, we adopt a composite communication network architecture of three level including WSN and Ethernet Passive Optical Network (EPON). The design involves the 
installation of a WSN of low cost, high monitoring precision, self-organization, and an EPON of high speed, high reliability, utilization of free OPGW cable cores. The network make full use of the optical fiber between substations and dispatching center for data transmission from substations to dispatching center. An array of sensor modules such as tension sensors, acceleration sensors, temperature sensors, infrared sensors and video images sensors are installed on each tower for information collection. Every tower is equipped with a relay node, which not only integrates received information but also send them to another nearby relay node that is closer to the optical fiber separated towers. Part of towers are optical fiber separated for fast delivery of gathering information. Our proposed communication network includes EPON network constructed by optical fiber separated towers, wireless communication of relay nodes and wireless communication between sensors and relay nodes.

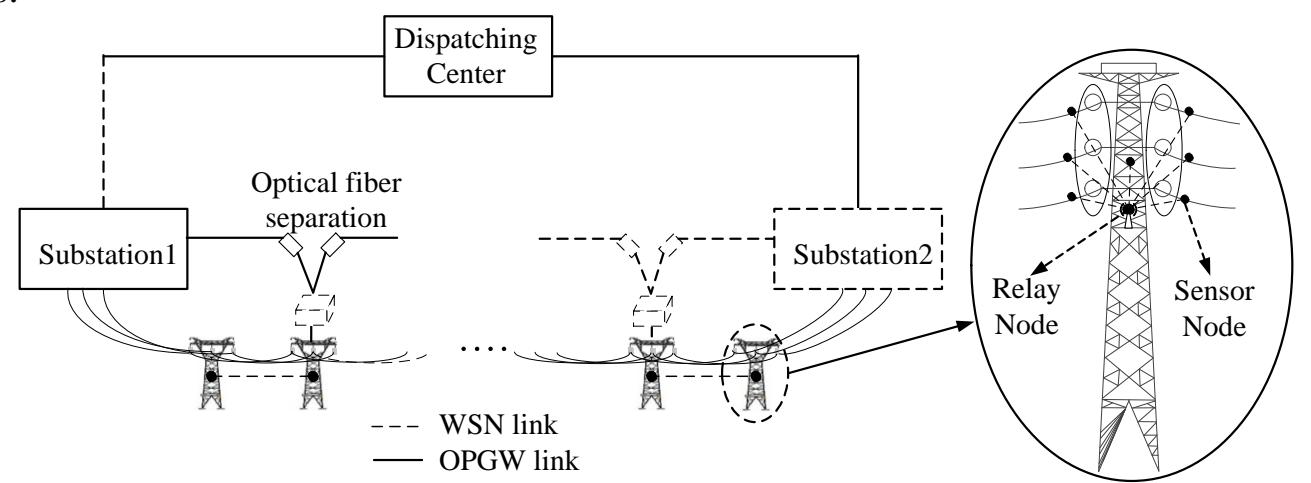

Fig. 1. Composite communication network architecture

An array of sensors on each tower form the first level. They collect information and send to the relay node of the tower. The second level consists of relay nodes on every tower. Relay nodes transmit monitoring information to optical fiber separated towers via nearby relay node in a multi-hop manner. The third level consists of optical fiber separated towers which constructed EPON. They are responsible for leading data into optical fiber and sending to substations.

B. Planning Formulation

On purpose of establishing a network with economic layout and real-time communication, the strategic placement of optical fiber separated towers becomes significant. Although optical fiber separated towers provide low latency, high bandwidth, it costs much to install. WSN devices are relatively inexpensive but provide lower data rate and bandwidth. In order to get the best tradeoff between cost and delay, we formulate a multi-objective placement problem to optimize the number of the optical fiber separated towers with constraint of bandwidth. In this problem, the network consists of $N$ transmission towers, two substations at the ends of transmission towers and a dispatching center. It costs $C_{\text {wsn }}$ yuan to install WSN devices on one tower and the arrangement cost of one optical fiber separated tower is $C_{\text {of }}$ yuan. $x_{i}$ is 1 only if tower $i$ is an optical fiber separated tower, which is a binary variables. $T_{\mathrm{CA}}$ denotes the average channel access latency of channel sense medium access/collision avoidance (CSMA/CA), and $m$ is the maximum hops went by data flows before reaching an optical fiber separated towers. Let $R_{\mathrm{d}}$ be the data rate of the relay node. $R$ denotes the transmission rate between two relay nodes. WSNB is the bandwidth of WSN links. The placement problem can be expressed as

Minimize:

$$
\begin{gathered}
f_{\text {cost }}=N \cdot C_{\text {wsn }}+\sum_{i=1}^{N} C_{\text {of }} x_{i} \\
f_{\text {latency }}=T_{\mathrm{CA}} \cdot m+\frac{R_{\mathrm{d}}\left(m^{2}+m-1\right)}{2 R}
\end{gathered}
$$

Subject to: 


$$
R_{\mathrm{d}} \bullet m \leq W S N B
$$

Our objective is to minimize the planning cost of composite communication network and the maximum end-to-end latency of data flows (hereinafter referred to as cost and latency) in the meantime as shown in Eq. 1. Our cost function is composed of two parts of installation cost of WSN devices on all transmission towers and arrangement cost of all optical fiber separated towers. Our latency function calculates the maximum end-to-end latency of data flow according to the transmission process of data. It involves the channel access latency related to CSMA/CA and the transmission latency related to data rate. Eq. 3 restricts the total bit rate of data flows through one link to less than the permitted bandwidth of WSN links.

\section{Optimization and Decision making Method}

The planning problem for composite communication network is a multi-objective optimization problem with characteristics of binary, multi-variable, discontinuous and nonlinear. In view of two inter-constraint objectives, we use Pareto optimal solution set to coordinate their relationship [7]. Particle Swarm Optimization (PSO) searches multiple solutions of the solution space in parallel by population operation. PSO is a kind of swarm intelligent optimization algorithm, whose procedures includes initialization, calculation of fitness value, update of particle velocity and position, update of optimal particle and non-inferior solution set.

On purpose of enhancing the search ability and avoiding premature convergence, we add adaptive inertia weight [8] and the global optimal position with random mutation [9] to traditional PSO. Adaptive inertia weight method adjusts the value of $w$ dynamically according to the difference of the current particle and the optimal particle. When the case of premature convergence happens, the random mutation of the global optimal position make it possible to search solutions continually in other areas of the solution space. The mutation probability of the optimal position is determined by the group fitness variance and the optimal solution. The mutation of the optimal position is realized by introducing random perturbation.

Based on the Pareto optimal solution set from PSO, multi-attribute decision making method is applied to select the most satisfactory solution. We firstly introduce triangular fuzzy number to reflect the subjective preference. The decision matrix, namely the Pareto optimal solution set, is used to calculate the objective weight by the information entropy method. The multi-attribute decision-making method based on fuzzy entropy weight of Vague set is used to sort Pareto solution set for the satisfaction solution.

Triangular fuzzy number is introduced to reflect the preference of decision makers to the cost attribute and delay attribute. We assume that the number of decision makers is $M$. The fuzzy weight vector of the $h$ th attribute is $\tilde{w}_{h}^{j}=\left(w_{h 1}^{j}, w_{h 2}^{j}, w_{h 3}^{j}\right)$ which is given by the $j$ th decision makers. Then the comprehensive fuzzy weight vector of the $h$ th attribute can be formulated as

$$
\tilde{w}_{h}=\frac{1}{M}\left(\tilde{w}_{h}^{1}+\tilde{w}_{h}^{2}+\cdots+\tilde{w}_{h}^{M}\right)=\left(\tilde{w}_{h 1}, \tilde{w}_{h 2}, \tilde{w}_{h 3}\right)
$$

The area center algorithm in Eq. 5 and normalization algorithm in Eq. 6 are used to calculate the fuzzy weight of the $h$ th attribute.

$$
\begin{gathered}
P_{h}=\frac{1}{3}\left(\tilde{w}_{h 1}+\tilde{w}_{h 2}+\tilde{w}_{h 3}\right) \\
w f_{h}=\frac{P_{h}}{\sum_{h=1}^{2} P_{h}}
\end{gathered}
$$

Information entropy weight method descript the variation degree of attributes by information entropy. So the objective weight of attributes is determined by the variation degree of the attribute in 
decision matrix. $\mathrm{B}=\left(b_{k h}\right)_{n \times 2}$ of is the decision matrix, where $n$ is the number of schemes, $b_{k h}$ is the cost or latency of the $k$ th scheme. Eq. 7 is used to normalize $b_{k h}$. The entropy value $H_{h}$ of the $h$ th attribute is obtained by Eq.8. Then Eq. 9 calculates the objective weight of the $h$ th attribute.

$$
\begin{gathered}
g_{k h}=\frac{\min b_{k h}}{b_{k h}}, \quad q_{k h}=\frac{g_{k h}}{\sum_{k=1}^{n} g_{k h}} 1 \leq k \leq n \\
H_{h}=-\frac{1}{\ln n}\left(\sum_{k=1}^{n} q_{k h} \ln q_{k h}\right) \\
w e_{h}=\frac{1-H_{h}}{\sum_{h=1}^{2}\left(1-H_{h}\right)}
\end{gathered}
$$

The fuzzy entropy of the $h$ th attribute is obtained by the weighted geometric mean method.

$$
z_{h}=\frac{w f_{h}-w e_{h}}{\sum_{h=1}^{2} w f_{h} \times w e_{h}}
$$

Vague set is a promotion form of fuzzy set. The decision procedures based on fuzzy entropy weight of Vague set are elaborated as follows.

Determining the membership degree vector of positive and negative ideal schemes, denoted as $g^{+}$, $g^{-}$.

$$
\left\{\begin{array}{l}
g^{+}=\left(g_{1}^{+}, g_{2}^{+}\right) \\
g^{-}=\left(g_{1}^{-}, g_{2}^{-}\right)
\end{array}\right.
$$

where $g_{h}^{+}=\max \left(g_{k h}\right), g_{h}^{-}=\min \left(g_{k h}\right)$, and $k=1,2, \cdots, n$.

The true and false membership degree of $g_{k h}$ to $g^{+}$and $g^{-}$respectively are calculated as

$$
\left\{\begin{array}{l}
t_{k h}^{+}=\frac{g_{k h}-g_{h}^{-}}{g_{h}^{+}-g_{h}^{-}} \quad t_{k h}^{-}=\frac{g_{h}^{+}-g_{k h}}{g_{h}^{+}-g_{h}^{-}} \\
f_{k h}^{+}=\frac{g_{h}^{+}-g_{k h}}{g_{h}^{+}-g_{h}^{-}} \quad f_{k h}^{-}=\frac{g_{k h}-g_{h}^{-}}{g_{h}^{+}-g_{h}^{-}}
\end{array}\right.
$$

Then the comprehensive Vague membership degree can be obtained by Eq. 13 .

$$
t_{k h}=t_{k h}^{+} f_{k h}^{-} \quad f_{k h}=f_{k h}^{+} t_{k h}^{-}
$$

Combining with the fuzzy entropy weight, the comprehensive Vague value $V_{k}=\left[t_{k}, f_{k}\right]$ of all schemes is computed.

$$
t_{k}=\sum_{h=1}^{2} z_{h} t_{k h} \quad f_{k}=\sum_{h=1}^{2} z_{h} f_{k h}
$$

Finally, we score and sort all schemes according to Eq. 15. Larger $S 1$ is superior to determine the better scheme and $S 2$ comes second.

$$
\left\{\begin{array}{l}
S_{1}=t_{k}-f_{k} \\
S_{2}=1-f_{k}
\end{array}\right.
$$




\section{Case Study}

We consider a transmission line with 40 towers. $R$ is $250 \mathrm{kbps}$ [10] and $R_{d}$ is $32 \mathrm{kbps}$ [2]. The installation cost WSN devices for each tower is 2000 Chinese yuan. It spends 120000 yuan to arrange an optical fiber separated tower on average based on investigation. $T_{\mathrm{CA}}$ is $41 \mathrm{~ms}$ [11]. We set the swarm size as 30 and run 600 iterations. The learning factor is 2.0. The initial value and end value of inertia weight are 0.9 and 0.4 respectively. The maximum and minimum mutation probability is 0.5 and 0. After simulation in MATLAB 2014a, we can get the Pareto optimal solution set as shown in Fig. 2.

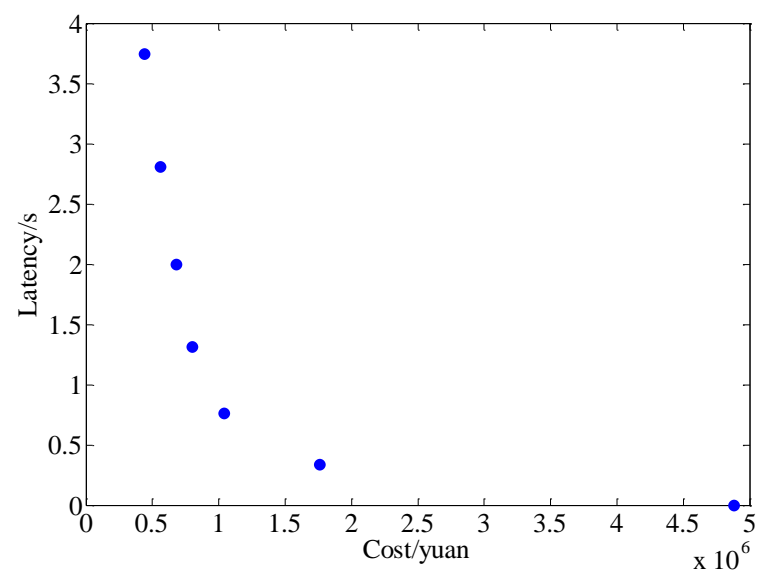

Fig. 2. Pareto optimal solution set

The solution with cost of 4.88 million yuan and latency of 0 s makes all towers optical-fiber-separated. Considering the practical use, we decide to wipe out this solution to avoid enormous cost and light attenuation. The fuzzy subjective weight vector is $[0.8211,0.1789]^{\mathrm{T}}$ according to the experiences of three network planning experts. The objective weight vector is $[0.1809,0.8191]^{\mathrm{T}}$ by using solution set. Thus the information entropy weight of latency is dominant. The fuzzy entropy weight vector is as $[0.5034,0.4966]^{\mathrm{T}}$ by calculation. Table 1 presents the scores of all schemes calculated by Eq. 11 15.

Table 1 Scoring results

\begin{tabular}{ccccc}
\hline Serial Number & Cost/10 $0^{4}$ yuan & Latency/s & Vague Score $(S 1, S 2)$ & Rank \\
\hline 1 & 176 & 0.338 & $(-0.043,0.479)$ & 2 \\
2 & 104 & 0.763 & $(-0.388,0.512)$ & 5 \\
3 & 80 & 1.316 & $(-0.408,0.493)$ & 6 \\
4 & 68 & 1.997 & $(-0.365,0.485)$ & 4 \\
5 & 56 & 2.806 & $(-0.224,0.510)$ & 3 \\
6 & 44 & 4.808 & $(0.043,0.521)$ & 1 \\
\hline
\end{tabular}

It is obvious that scheme 6 is the best solution. Scheme 6 has the lowest cost and the largest latency. What' more, the latency satisfies the transmission requirement of SCADA system [12]. It can be seen from the optimization of PSO and multi-attribute decision making process that our solving method avoid the blindness of linear weighted method and obtain uniform solutions which provide a broader choice space. Fig. 2 indicates that the two inter-constraint objectives are unlikely to be optimized simultaneously. So we can only get the most satisfactory composite communication network layout scheme from alternatives according to the actual layout requirements and the preference information of decision makers.

\section{Analysis on the Influence Factors of Placement}

For anyone certain transmission corridor, a scientific and reasonable solution can be obtained by above method. On the basis of the above parameters, we study several different scenarios including variation in subjective weight, data rate and network size. 


\section{A. Effect of Subjective Weight}

The value of subjective weight will influence the decision of placement schemes. But there is no specification about the balance between cost and delay and the value of subjective weight depends on the past project experience of decision makers. Fig. 3 shows the effect of variation in the subjective weight of the two attributes with respect to the decision of placement schemes.

When the subjective weight of cost is less than 0.8191, scheme 1 is the best placement; when it is greater than 0.8191, scheme 6 is the best placement; and when it lies at critical point, the fuzzy entropy weight of the two attributes is 0.5 and the score of scheme 1 and 6 is the same. Therefore, different subjective weight will result in two different optimal scheme. In addition, the simulation test result indicates the variable subjective weight will change the priority order of schemes.

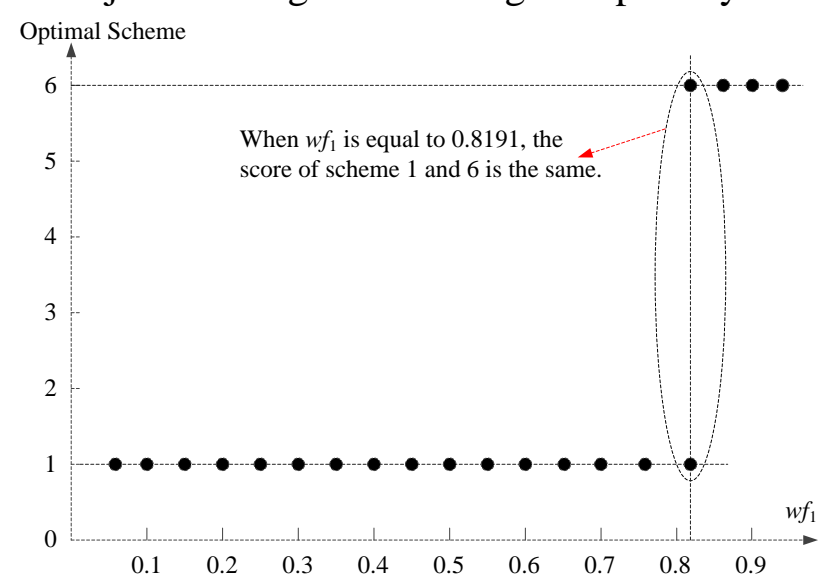

Fig. 3 Impact of subjective weight on scheme decision making

\section{B. Effect of Flow Data Rate}

Both flow data rate and limited link bandwidth determine the number of data flow in wireless links and the farthest tower whose data can be collected by an optical fiber separated tower. They certainly have an impact on the arrangement of communication network. Table 2 shows the effect of variation in data rate with respect to the number of alternatives and the best scheme.

When flow data rate is less than $128 \mathrm{kbps}$, the number of alternatives corresponding to every kind of flow data rate is different. What's more, the bigger flow data rate is, the fewer the number of alternatives is. When flow data rate is greater than or equal to $128 \mathrm{kbps}$, there is only one alternative. Because the bandwidth limit is $250 \mathrm{kbps}$, one data flow is allowed to pass in each link at most. If flow data rate goes over $250 \mathrm{kbps}$, it is necessary to make every tower optical fiber separated. In addition, with fewer alternatives, the advantage of latency in information entropy becomes weaker so that the fuzzy entropy weight has a positive bias toward cost and the best scheme tends to be the scheme with lower cost.

\begin{tabular}{cccc}
\multicolumn{4}{c}{ Table 2 Impact of flow's bit rate on scheme decision making } \\
\hline Flow Data Rate & Alternatives & Best Scheme & Fuzzy Entropy Vector \\
\hline 32 & $6(1 \sim 6)$ & 6 & {$[0.5034,0.4966]^{\mathrm{T}}$} \\
48 & $5(1 \sim 5)$ & 5 & {$[0.5065,0.4935]^{\mathrm{T}}$} \\
56 & $4(1 \sim 4)$ & 4 & {$[0.5284,0.4716]^{\mathrm{T}}$} \\
64 & $3(1 \sim 3)$ & 3 & {$[0.5396,0.4604]^{\mathrm{T}}$} \\
96 & $2(1,2)$ & 2 & {$[0.6438,0.3562]^{\mathrm{T}}$} \\
128 & 1 & 1 & - \\
160 & 1 & 1 & \\
192 & 1 & 1 & \\
\hline
\end{tabular}

C. Effect of Network Size

Table 3 summarizes the number of alternatives and the best scheme for different number of towers. The specific alternatives are show in Table 4. When the number of towers is small (40-60), feasible 
solutions with different latency can have the same number of optical fiber separated tower. In this case, the solution with smaller latency will be chosen and the number of alternatives will be less than 7 . It no longer happens with the increase in the network size. The variation of the best scheme decision under different number of towers is influenced by not only the number of alternatives but also the value of cost and latency of schemes.

Table 3 Impact of the number of towers on scheme decision making

\begin{tabular}{cccc} 
Number of & Alternatives & \multicolumn{2}{c}{ Best Scheme } \\
\cline { 3 - 4 } Towers & Cost/yuan & Latency/s \\
\hline 20 & 4 & $28 \times 10^{4}$ & 2.806 \\
30 & 6 & $30 \times 10^{4}$ & 4.808 \\
40 & 6 & $44 \times 10^{4}$ & 4.808 \\
50 & 6 & $58 \times 10^{4}$ & 3.743 \\
60 & 7 & $252 \times 10^{4}$ & 0.338 \\
80 & 7 & $340 \times 10^{4}$ & 0.338 \\
100 & 7 & $428 \times 10^{4}$ & 0.338 \\
120 & 7 & $504 \times 10^{4}$ & 0.338 \\
150 & 7 & $630 \times 10^{4}$ & 0.338 \\
\hline
\end{tabular}

Table 4 Alternative schemes of scenes with different number of towers

\begin{tabular}{cc}
\hline Number of Towers & Alternatives $($ Cost/10 4 yuan, Latency/s) \\
\hline 20 & $(88,0.338),(52,0.763),(40,1.316),(28,2.806)$ \\
30 & $(126,0.338),(78,0.763),(66,1.316),(54,1.997),(42,2.806),(30,4.808)$ \\
40 & $(176,0.338),(104,0.763),(80,1.316),(68,1.997),(56,2.806),(44,4.808)$ \\
50 & $(214,0.338),(130,0.763),(106,1.316),(82,1.997),(70,2.806),(58,3.743)$ \\
60 & $(252,0.338),(156,0.763),(120,1.316),(96,1.997),(84,2.806),(72,3.743),(60,4.808)$ \\
80 & $(340,0.338),(208,0.763),(160,1.316),(124,1.997),(112,2.806),(100,3.743),(88,4.808)$ \\
100 & $(428,0.338),(260,0.763),(200,1.316),(164,1.997),(140,2.806),(116,3.743),(104,4.808)$ \\
120 & $(504,0.338),(312,0.763),(240,1.316),(192,1.997),(156,2.806),(144,3.743),(120,4.808)$ \\
150 & $(630,0.338),(390,0.763),(294,1.316),(234,1.997),(198,2.806),(174,3.743),(150,4.808)$ \\
\hline
\end{tabular}

\section{Conclusions}

In this paper, we presented an optimal formulation for a cost and latency optimized composite communication network design capable of transmission of sensor data through the transmission line network in the presence of bandwidth constraint. A simulation case and the influence factors of placement are analyzed, which helps electric power developer to balance efficiency and economy for scientific and reasonable communication network planning. As part of future work, we plan to study the composite communication architecture introducing Wireless Fidelity.

\section{Acknowledgements}

This work was financially supported by Technology and National High Technology Research and Development Program (NO. 2015AA050201).

\section{References}

[1] V. C. Gungor, B. Lu, and G. P. Hancke. "Opportunities and challenges of wireless sensor networks in smart grid - A case study of link quality assessments in power distribution systems," IEEE Trans. Ind. Electron., vol. 57, no. 10, pp. 3557-3564, 2010. 
[2] K. S. Hung, W. K. Lee, V. O. K. Li, et al., "On wireless sensors communication for overhead transmission line monitoring in power delivery systems," in Proc. IEEE International Conference on Smart Grid Communications, Gaithersburg, 2010, pp. 309-314.

[3] J. Ausen, B. F. Fitzgerald, E. A. Gust, et al., "Dynamic thermal rating system relieves transmission constraint," in Proc. IEEE 11th International Conference on Transmission \& Distribution Construction, Operation and Live-Line Maintenance, Albuquerque, 2006, pp. 1-5.

[4] Y. H. Zhu, Y. Luo, T. Duan, et al., "Online risk assessment based on real-time evaluation model of transmission line for static security of power system," Electric Power Automation Equipment, vol. 34, no. 7, pp. 150-156, 2014.

[5] Y. C. Wu, L. F. Cheung, K. S. Lui, et al., "Efficient Communication of sensors monitoring overhead transmission lines," IEEE Transactions on Smart Grid, vol. 3, no. 3, pp. 1130-1136, 2012.

[6] B. Fateh, M. Govindarasu, and V, Ajjarapu. "Wireless network design for transmission line monitoring in smart grid," IEEE Transactions on Smart Grid, vol. 4, no. 2, pp. 1076-1086, 2013.

[7] A. M. Zhou, Q. F. Zhang, and Y. C. Jin, "Approximating the Set of Pareto-Optimal Solutions in Both the Decision and Objective Spaces by an Estimation of Distribution Algorithm," IEEE Transactions on Evolutionary Computation, vol. 13, no. 5, pp. 1167-1189, 2009.

[8] M. Y. Chen, C. S. Wu, and P. Fleming, "An evolutionary particle swarm algorithm for multi-objective optimization," in Proc. 7th World Conference on Intelligent Control and Automation, Chongqing, 2008, pp. 3269-3274.

[9] J. Q. Yang, J. H. He, X. J. Wang, and Z. Q. Bo, "Optimal Control Strategy of Electric Vehicles Based on the Adaptive Mutation of PSO Algorithm," in Proc. Asia-Pacific Power and Energy Engineering Conference, Shanghai, 2012, pp. 1-5.

[10]IEEE Standard for Information Technology-Part 15.4: Wireless Medium Access Control (MAC) and Physical Layer (PHY) Specifications for Low Rate Wireless Personal Area Networks (LRWPANS), IEEE Std 802.15.4-2006.

[11] V. Shnayder, M. Hempstead, B. Chen, et al., "Simulating the power consumption of large-scale sensor network applications," in Proc. 2nd International Conference on Embedded Networked Sensor Systems, Baltimore, 2004, pp. 188-200.

[12] IEEE Standard for SCADA and Automation Systems, IEEE Std C37.1-2007. 